\title{
Criminologie
}

\section{Nouveaux courants en criminologie : " études sur la justice " et " zémiologie "}

\section{Marie-Andrée Bertrand}

Volume 41, numéro 1, printemps 2008

40 ans de Criminologie : perspectives d'avenir

URI : https://id.erudit.org/iderudit/018424ar

DOI : https://doi.org/10.7202/018424ar

Aller au sommaire du numéro

\section{Éditeur(s)}

Les Presses de l'Université de Montréal

ISSN

0316-0041 (imprimé)

1492-1367 (numérique)

Découvrir la revue

Citer cet article

Bertrand, M.-A. (2008). Nouveaux courants en criminologie : « études sur la justice » et " zémiologie ». Criminologie, 41(1), 177-200.

https://doi.org/10.7202/018424ar
Résumé de l'article

S'appuyant sur trois bilans assez négatifs de la discipline dressés par des criminologues canadiens connus, l'auteure de cet article analyse les conditions dans lesquelles est née la criminologie afin d'éclairer ce qui rend si difficile la critique de fond de son objet, le crime. Les experts invoquent le caractère appliqué et normatif de la discipline, sa proximité avec le pouvoir politique, ainsi que le climat social et politique, national et international, favorable à un retour aux orientations répressives et à une gestion actuarielle du pénal. Mais comment expliquer alors que des groupes de criminologues britanniques et irlandais, vivant dans un contexte social et politique analogue à celui des Canadiens, aient réussi à se tailler un objet d'étude au-delà du droit pénal, et qu'au Canada même une commission nationale du droit en arrive à questionner radicalement l'objet de la criminologie et à se rallier à la notion de " tort social grave " comme sujet d'étude et d'intervention ? L'auteure suggère que la professionnalisation de la discipline au Canada rend difficile toute critique immanente. Malgré tout, elle voit des signes d'un possible renouvellement de la criminologie dans l'intérêt que manifestent des départements pour les « Justice Studies » et dans le mouvement amorcé par l'Association canadienne de criminologie et sa revue qui ajoutent le mot " justice " à leur nom. 


\title{
Nouveaux courants en criminologie: « études sur la justice » et « zémiologie »
}

\author{
Marie-Andrée Bertrand \\ Professeure émérite, École de criminologie, Université de Montréal \\ Chercheure, Centre international de criminologie comparée \\ bertrandumontreal@videotron.ca
}

RÉSUMÉ - S'appuyant sur trois bilans assez négatifs de la discipline dressés par des criminologues canadiens connus, l'auteure de cet article analyse les conditions dans lesquelles est née la criminologie afin d'éclairer ce qui rend si difficile la critique de fond de son objet, le crime. Les experts invoquent le caractère appliqué et normatif de la discipline, sa proximité avec le pouvoir politique, ainsi que le climat social et politique, national et international, favorable à un retour aux orientations répressives et à une gestion actuarielle du pénal. Mais comment expliquer alors que des groupes de criminologues britanniques et irlandais, vivant dans un contexte social et politique analogue à celui des Canadiens, aient réussi à se tailler un objet d'étude au-delà du droit pénal, et qu'au Canada même une commission nationale du droit en arrive à questionner radicalement l'objet de la criminologie et à se rallier à la notion de «tort social grave» comme sujet d'étude et d'intervention? L'auteure suggère que la professionnalisation de la discipline au Canada rend difficile toute critique immanente. Malgré tout, elle voit des signes d'un possible renouvellement de la criminologie dans l'intérêt que manifestent des départements pour les «Justice Studies» et dans le mouvement amorcé par l'Association canadienne de criminologie et sa revue qui ajoutent le mot «justice» à leur nom.

ABSTRACT - Experts in the field agree that criminology has shown its lack of capacity to examine critically its object, crime, as defined in penal law and see four factors that have contributed to these lacunae, the applied and normative character of the discipline, its proximity to political power, the national and international social climates that favour penal repression and actuarial management rather than intellectual enlightenment. But then, asks the author, how to explain that under similar social and political conditions, a group of British and Irish social scientists have emancipated themselves from the discipline and chosen a larger field of study, beyond criminology as they say? And how was it possible for the Canadian Law Commission to explore the 
radical question "What is a crime?" and to put twelve researchers at work on case studies that demonstrate the incapacity of penal law to apprehend and control severe social harms, rejoining the British and Irish colleagues' move? Is the professionalization of the discipline responsible for criminology's difficulty to "think critically" ? The author sees a glimmer of hope in the will to change name shown by some criminology departments, a small move that the Canadian Association and Journal of Criminology have already made, adding the word "justice" to their title.

\section{Introduction}

On ne remerciera jamais assez les collègues de l'École de criminologie de Montréal qui ont bien voulu diriger la revue Criminologie et en assurer la continuité malgré les caprices des bailleurs de fonds et les exigences contradictoires des organismes de subvention depuis quarante ans. Il n'est pas facile de démontrer empiriquement ce qui aurait manqué à la criminologie d'expression française si Criminologie n'avait pas existé ou si sa parution avait été interrompue pendant de longues périodes. Ces hypothèses malheureuses ne s'étant pas avérées, il existe une autre façon d'apprécier l'effet de la présence de la revue: les liens entre générations de professeurs et d'étudiants à l'École de Montréal sont étroits et chacun ou presque sait que, sans l'accueil fait par la revue à ses travaux, à ceux de ses collègues et de ses doctorants, l'entrée et l'avancement dans la carrière auraient été difficiles.

\section{L'objet de cet article}

À l'instar des auteurs qui, en 1986, ont contribué au numéro spécial de la revue Criminologie marquant le $25^{\mathrm{e}}$ anniversaire du périodique ${ }^{1}$, je traiterai dans cet article de la discipline et de son orientation à partir des questions suivantes: quelle place occupe la pensée critique dans le discours savant des criminologues au Québec et Canada, mais surtout quelle importance la criminologie canadienne accorde-t-elle à la notion de «crime» dans son discours et ses pratiques? Mes hypothèses sont que la criminologie canadienne a en général fait peu de place à la

1. Voir la contribution de Pierre Landreville (1986), «Évolution théorique en criminologie, l'histoire d'un cheminement», Criminologie, XIX(1), 11-31. Voir aussi dans le même numéro thématique l'analyse des courants théoriques que leurs auteurs reconnaissent comme prometteurs, par exemple l'analyse stratégique (Maurice Cusson, 53-71), l'approche intégrative (Marc LeBlanc, 73-95), les perspectives critiques (Marie-Andrée Bertrand, 97-111). 
pensée critique et encore moins à la réflexion sur la notion de crime. Ses experts travaillent en aval, c'est-à-dire sur les conséquences de l'application du droit criminel plutôt qu'en amont, ils mettent leurs énergies et leurs savoirs au service des personnes criminalisées et du bon fonctionnement du système pénal dont ils s'efforcent de rendre l'application la plus juste et la plus humaniste possible.

Mon intérêt pour la théorie critique et mon engagement dans les mouvements qui s'en inspirent ne sont pas nouveaux (Bertrand, 1986; 1999 ; $2000 ; 2004)$. Sur la notion juridique de crime, j'entretiens un débat constant avec le droit pénal depuis quarante ans $(1967$; 1999b; 2003); cet intérêt s'est encore accru avec ma participation aux travaux de la Commission du droit du Canada depuis 2002 sur le thème «What is a Crime? ». Dans la discipline elle-même, des signes d'intérêt en faveur d'un renouvellement des perspectives et des pratiques sont apparus ou réapparus depuis 1999 au Canada et ailleurs, allant dans le sens de mes préoccupations. C'est à la description de ces tendances que je m'emploie dans cet article. Puisque cette livraison spéciale de la revue Criminologie est l'occasion d'évoquer plusieurs anniversaires, ceux de la naissance de la discipline (1960), de la revue (1968), et du Centre international de criminologie comparée (1969), j'ai cru pertinent de rappeler les conditions sociales, intellectuelles et institutionnelles qui existaient au début des années 1960, puis de résumer le jugement des collègues sur l'état des lieux. Ce n'est qu'après ce portrait global de la scène canadienne que j'expose les nouveaux courants tels qu'ils se manifestent dans les Justice Studies et la zémiologie et que j'évoque l'orientation des travaux de la Commission du droit du Canada.

\section{La criminologie canadienne}

\section{L'École de Montréal}

L'École de criminologie de l'Université de Montréal célébrera bientôt son cinquantième anniversaire, un demi-siècle d'enseignement et de recherche. Sans nier le caractère original de l'institution de Montréal et le rôle particulier joué par Denis Szabo dans la création du Département, il faut rappeler que le mot lui-même, «criminologie», a connu des usages variés avant 1960, qu'une telle initiative ne fut pas la seule du genre ni la première en Amérique du Nord, enfin, que la naissance d'une «nouvelle» criminologie dans les années 1960 n'était possible 
qu'à un moment où les sciences sociales connaissaient un essor remarquable.

D'abord, les usages du terme «criminologie». En 1960, quand naissent l'Institut de Cambridge et le Département de criminologie de Montréal, existent déjà depuis plusieurs années un bon nombre d'écoles et de collèges qui dispensent, sous le label "criminology", des enseignements en sciences policières et en criminalistique allant jusquau doctorat: c'est le cas notamment des universités des États de Floride, du Michigan et du Maryland. En Europe continentale, on utilise aussi le mot «criminologie», mais dans ce cas pour désigner des diplômes dispensés par les facultés de droit aux policiers, aux procureurs, aux juges d'instruction: on appelle aussi «criminologiques» les formations en médecine et en psychiatrie légale.

La «nouvelle» criminologie qui naît dans les universités de Cambridge, Montréal, Berkeley et Toronto au début des années 1960 est tout autre chose. C'est une discipline qui se nourrit des sciences sociales, du droit et de quelques sciences humaines comme la psychologie, mais aussi parfois des sciences de la santé comme la psychiatrie; cette criminologie offrira des programmes d'études et des grades aux trois cycles, généralement dans les facultés de sciences sociales, mais parfois aussi dans les facultés de droit.

Un mot sur ces naissances simultanées. Entre 1960 et 1963, la «nouvelle» criminologie naît concomitamment dans trois pays: en 1960 en Angleterre à l'Institut de criminologie de l'Université de Cambridge $^{2}$ et au Canada au Département de criminologie de l'Université de Montréal qui offre un programme de maîtrise ${ }^{3}$, et en 1961, à l'École de criminologie de l'Université de Californie, Berkeley, laquelle crée un baccalauréat général et une maîtrise, puis un doctorat en 1963. À Berkeley, les programmes d'étude portent comme ceux de Montréal sur le crime, le criminel et la politique pénale, mais s'y ajou-

2. L'Institut de criminologie de l'Université de Cambridge a été fondé en 1960 par Sir Leon Radzinowicz, c'était le premier en Europe et il a exercé une grande influence sur le développement de la discipline.

3. Sur la naissance du programme de Maîtrise en criminologie à l'Université de Montréal, voir l'Éditorial du numéro spécial de Criminologie, XIX (1), 3. Voir aussi dans Denis Szabo, Fondation et fondements de la criminologie, par Marcel Fournier $(1998,90)$ concernant les premiers cours de niveau maîtrise offerts à l'Université de Montréal. Avant 1960, une introduction générale à la criminologie a été dispensée par Denis Szabo en cours du soir. 
tent des cours en sciences administratives ${ }^{4}$ et l'École continue d'offrir des enseignements en sciences policières, ce qu'elle faisait depuis 1920. En 1963, au Canada, John Edwards crée le Centre de criminologie de l'Université de Toronto, d'abord essentiellement dédié à la recherche sous l'égide de la faculté de droit ${ }^{5}$; les enseignements viendront plus tard, dans des programmes de $2^{\mathrm{e}}$ et de $3^{\mathrm{e}}$ cycle seulement ${ }^{6}$.

Bref, la criminologie est tout sauf une «monodiscipline». Alors que son intitulé fait appel à un concept juridique, le crime, elle compose ses programmes d'études en fonction de facteurs historiques et institutionnels.

Il est important de connaitre les conditions de la naissance de cette criminologie canadienne qui se nourrit des sciences sociales et humaines pour comprendre les orientations qu'elle prendra. Ces champs de savoir et de savoir-faire sont en pleine expansion au moment où naît la criminologie. Dans les universités anglophones et les pays de common law, ils prennent alors un virage d'une importance capitale. Quel virage? Dans les années 1960, on assiste à une démocratisation sans précédent de l'enseignement postsecondaire et à une explosion des populations universitaires dans les pays occidentaux; les sciences sociales et humaines n'y échappent pas, au contraire. Disciplines nouvelles, auxquelles plusieurs nient le statut de «sciences», elles sont fortement incitées, pour ne pas dire contraintes, par les ministères responsables de l'enseignement supérieur et par la direction des établissements, à rendre leurs programmes accessibles à toutes les clientèles possibles et, surtout, à s'occuper activement de faire « une science utile» qui pourra «résoudre»

4. Un programme de formation en sciences policières et criminalistique était offert depuis 1920 à Berkeley. L'Université créera un Bureau de criminologie en rapport avec le programme de science politique, puis une École (autonome) en 1950 offrant des cours sur les facteurs du crime, la criminalistique et les établissements correctionnels.

5. «Fondé par John Edwards en 1963, le Centre de criminologie de Toronto regroupe des professeurs et des étudiants qui étudient le crime, l'ordre social, la sécurité, depuis plusieurs perspectives disciplinaires et approches théoriques. Formés dans plusieurs disciplines, la sociologie, le droit, la psychologie, la philosophie et la science politique, les professeurs s'impliquent dans la recherche en criminologie, au Canada et dans le monde international». Ma traduction. Source: http://criminology.utoronto.ca/index.html

6. Aux États-Unis, le «modèle mixte», sciences sociales et sciences de la détection du crime, perdure dans plusieurs centres universitaires. Au Canada, les savoirs dispensés ressortissent aux sciences humaines et sociales et au droit; des programmes de $1^{\text {er }}$ et de $2^{\mathrm{e}}$ cycle sur la sécurité et la police se développent à Montréal, et des centres de psychiatrie médicolégale se taillent une place importante dans le voisinage de «la criminologie de sciences sociales» à Toronto et à Simon Fraser. 
les problèmes sociaux, politiques, économiques, à offrir des enseignements «pratiques» et à enseigner des pratiques (Clark, 1998; Geiger, 2004). Elles doivent non seulement faire la preuve de leur intelligence des questions qu'il faut poser à la science, mais aussi démontrer leur capacité d'appliquer leurs connaissances à la résolution des problèmes concrets. Désormais, il leur faut comprendre et expliquer, trouver et appliquer des solutions, corriger, réformer. Même les disciplines fondamentales comme la sociologie, l'anthropologie, l'économie, et plus tard la science politique, vont devoir se donner des champs d'intervention. Les pressions externes et internes se feront plus nombreuses dans les disciplines appliquées comme le service social, la démographie, les relations industrielles, la criminologie, les sciences de l'éducation et celles de l'administration.

Dans cette conjoncture, c'est la criminologie qui sera soumise aux contraintes les plus nombreuses : étendre rapidement ses enseignements au premier cycle pour attirer les clientèles; prouver son utilité sociale et politique; développer des champs d'application; former des intervenants qui exerceront leur métier avec des diplômes de premier cycle; provoquer la naissance de nouveaux secteurs d'intervention et d'emplois; développer concomitamment des centres de recherche pour asseoir la réputation scientifique de la discipline, si ce n'était déjà fait, ou accélérer la création d'enseignements là où la criminologie s'était d'abord définie comme centre de recherche; enfin, former des experts, dont l'État requerra les services lors de ses grandes enquêtes sur le droit pénal et correctionnel, et développer des compétences en matière de politiques pénales. On relira avec intérêt les entrevues accordées par Denis Szabo à Marcel Fournier sur ce sujet (Fournier, 1998); en voici un court extrait:

Je voyais haut et loin, bien au-delà du service social. Je visais la création d'une école professionnelle dotée d'une forte composante de recherche, regardant au-delà des frontières disciplinaires et politiques. C'est à ce moment que j’ai créé le CICC. (Fourier, 1998: 105)

Le mot est lancé: une école professionnelle en sciences sociales appliquées. Comment l'École de criminologie de Montréal s'est-elle acquittée de cette tâche titanesque?

Pas toujours très bien. Le bilan des 25 premières années que tracent mes collègues dans le numéro thématique de la revue Criminologie est mitigé. Sur le plan théorique, selon Landreville (1986: 11-31), l’École 
s'est d'abord centrée sur la conduite criminelle et le délinquant et s'est lancée dans une entreprise de réforme des criminels, pour ensuite reconnaître que les traitements et les programmes n'avaient pas d'effet sensible sur le taux de récidive. Dans un deuxième temps, durant les années 1970 à 1980, l'influence de la sociologie s'est fait sentir avec l'engagement de nouveaux professeurs: on a reconnu le poids des institutions sociales et pénales dans la criminalisation des personnes et des groupes sociaux. Puis, on s'est intéressé aux mécanismes de contrôle de la criminalité et les perspectives théoriques se sont déplacées du positivisme biologique ou psychologique vers le constructivisme, l'interactionnisme et la théorie de la réaction sociale. Mais à l'École de criminologie de Montréal, on est peu marqué par les perspectives critiques qui, à la même époque pourtant, transforment littéralement les enseignements et la recherche à l'École de Berkeley ${ }^{7}$; l'écart est encore plus grand avec les Britanniques dont les deux traités sur la criminologie critique et la criminologie nouvelle auront une grande influence dans les pays anglophones (Australie, États-Unis), et même dans les pays scandinaves (voir Bertrand, 1986: 97-112). Quant aux perspectives abolitionnistes, elles sont accueillies avec sympathie par quelques chercheurs influents à l'École de Montréal, mais rejetées comme utopistes par la majorité. À la longue cependant, elles marqueront les positions de plusieurs criminologues canadiens, anglophones et francophones, souvent appelés à conseiller les décideurs et à siéger aux grandes commissions d'enquête fédérales ou provinciales sur la justice, la police, les drogues et les prisons. La position canadienne en matière de politiques pénales, surtout celle des criminologues québécois, sera décrite comme «minimaliste, humaniste et libérale ${ }^{8} »$, privilégiant la

7. J'ai été témoin de cette transformation. Étudiante au doctorat de 1965 à 1967 dans cette école de criminologie libérale mais ouverte à la critique depuis le Free Speech Movement de 1964, j'ai été invitée à y enseigner en 1972, alors que l'influence des marxistes radicaux, les époux Schwendinger, Anthony Platt, Dorie Klein et quelques autres, se faisait fortement sentir. La politisation des professeurs et des étudiants était évidente et la réaction ne s'est pas fait attendre. Le chancelier de l'époque, Ronald Reagan, ordonna la fermeture de l'École en 1977.

8. En 2007, un professeur de droit pénal et de criminologie de l'Université de Brême, Johannes Feest, a servi de guide aux étudiants en criminologie de Hambourg et de Brême qui voulaient faire le point sur l'abolitionnisme. Il a lancé un dialogue en ligne sur la question: «Does Abolitionism have a Future?». Une vingtaine de participants parmi lesquels se trouvent les plus célèbres abolitionnistes des pays du Nord de l'Europe, René van Swaaningen, Nils Christie, Thomas Mathiesen, etc. ont participé à cette conversation. En général, la réponse est la suivante: l'abolitionnisme (des prisons surtout) a été un 
réduction des chefs d'accusation, moins de peines carcérales et plus de solutions de rechange.

Par ailleurs, l'École de Montréal innovera en victimologie, et elle ne cessera de prôner et de pratiquer une justice des mineurs plus équitable, plus éducative et plus communautaire qu'ailleurs (Trépanier, 1986). En recherche, les études sur la police (Brodeur, 1983; 1986), sur les prisons (Landreville, 1986; Lemire, 1986; Normandeau, 1986), sur le traitement pénal des femmes (Bertrand, 1998) sont à la fois critiques du statu quo et réformistes.

Les tendances critiques déjà à l'œuvre dans les années 1980 s'expriment avec une grande force à la fin des années 1990 dans deux champs particuliers: d'abord celui de la police notamment avec les travaux de Brodeur sur le "high and low policing" (1983) ${ }^{9}$. Cette distinction est utilisée par les responsables de la sécurité nationale en période de terrorisme pour légitimer les interrogatoires s'apparentant à la torture et pour maintenir en détention des personnes innocentes tant qu'elles n'auront pas livré les informations attendues. Tout cela va à l'encontre des chartes et même du droit pénal, sous le regard approbateur de l'État. Une deuxième forme de critique radicale est pratiquée par Jaccoud (2003), qui choisit comme champs de recherche et d'enseignement les solutions de rechange au contrôle pénal, la médiation et la réparation, des pratiques s'inspirant des traditions autochtones de règlement des conflits.

\section{Dans l'ensemble du Canada}

La criminologie canadienne hors Québec évolue-t-elle différemment? Oui et non, comme le démontrent deux ouvrages collectifs, l'un datant de 1999 et l'autre de 2006.

mouvement, une pratique, une pensée, et sous ces trois formes il a un avenir, différent et plus large que son passé. Cette conversation sera publiée dans le Kriminologisches Journal du Département de sciences sociales de l'Université de Hambourg en 2008. Invitée à participer à cette conversation, j'ai décrit les liens entre les centres canadiens de criminologie, francophones et anglophones, et les leaders du mouvement abolitionniste, et les effets de ces contacts sur la criminologie canadienne. Prenant appui sur mon exposé écrit, Johannes Feest décrit la criminologie canadienne comme inspirant des politiques pénales «humanistes, libérales et minimalistes».

9. Un autre article de Jean-Paul Brodeur, non encore publié, porte sur le même sujet; l'auteur m'en ayant communiqué le contenu, j'en évoque ici les idées clés. 
L'état de la recherche (1999)

Le bilan que dessinent les auteurs ayant contribué en 1999 au numéro thématique de la Revue canadienne de criminologie (41 [2]) est plutôt négatif: plusieurs analystes sont déçus des réalisations antérieures, sauf en ce qui concerne les années 1970 à 1985, et pessimistes quant à l'avenir. D'autres partagent un optimisme modéré, tandis que deux contributions décrivent des contextes propices à la production intellectuelle.

Parmi les optimistes, on trouve les collègues de l'Université Simon Fraser qui se réjouissent de la croissance exponentielle de leur département qui forme des chercheurs féconds et nombreux; selon eux, l'avenir de la recherche est assuré (Menzies et Chunn, 1999); deux chercheurs de Montréal en arrivent aux mêmes conclusions pour les mêmes raisons, chiffres à l'appui (Normandeau et Cusson, 1999). De leur côté, les historiens du droit pénal ne sont pas trop malheureux, car les progrès des moyens de communications permettent de poursuivre les travaux entrepris avec des ressources modestes et ils arrivent à en trouver (Smandych et Hogeveen, 1999). D'autres estiment que leur champ de recherche, la justice des mineurs et les services correctionnels, est relativement épargné en l'occurrence (Doob, 1999; Roberts, 1999). Trois auteures se réjouissent des nouvelles perspectives dans lesquelles s'engage la recherche dans leur domaine, ce sont des féministes, heureuses que leur courant théorique s'ouvre à la diversité (Comack, 1999); malheureusement, on ne voit pas bien ce qu'apportera cette diversité à elle seule (la reconnaissance des problèmes qu'éprouvent les hommes devant les nouvelles postures féminines?). Deux autres estiment que les théories de la modernité avancée, l'analyse du discours et la transdisciplinarité, s'éloignant de la rationalité moderne, constituent des progrès (Stenning et Murphy, 1999). Tous ceux-là représentent le groupe des satisfaits et des «rassurés».

Pour ma part, je ne vois rien de rassurant dans ces dernières tendances. On ne comprend pas bien pourquoi la fragmentation proposée aurait des vertus innées et des conséquences positives.

Ailleurs, l'inquiétude domine. Notons tout d'abord que cette livraison thématique paraît peu après que les ministères fédéraux de la Justice, du Solliciteur général et des Établissements correctionnels aient décidé de mettre fin aux subventions de l'État à la recherche en criminologie. Non seulement c'est la fin des contrats individuels entre ministères et chercheurs, mais on va voir se tarir les sources de subvention aux 
centres canadiens de recherche en criminologie (si les États provinciaux ne prennent pas la relève, ce qu'ils n'ont pas tous fait).

Mais l'inquiétude d'un bon nombre d'auteurs porte sur un autre objet, sept d'entre eux s'interrogent sur «la performance de la discipline» et les sujets mêmes des études subventionnées jusque-là par l'État: ils notent que dans les temps d'abondance et de subventions généreuses, la recherche en criminologie a été très politisée (Brodeur, 1999; Clairmont, 1999; Woods, 1999; Stenning, 1999; Murphy, 1999; Laprairie, 1999; Wood et Shearing, 1999). Les services de la recherche des ministères de la Justice et des Services correctionnels ont utilisé leur budget pour dicter les sujets utiles aux yeux du gouvernement ou des hauts fonctionnaires. Chez certains auteurs, l'humeur est au noir. Comme le rappelle Brodeur (1999), en temps d'abondance comme en période de disette, la recherche universitaire a peu d'influence sur les décideurs; il n'est pas rare que les décisions aillent directement à l'encontre des résultats de recherche les plus clairs et unanimes. C'est le cas notamment des sentences fixes dont l'inutilité est bien démontrée. Devenant presque cynique, Brodeur insiste: dans les années 1970-1980, la criminologie a joué un rôle important de démystification des lieux communs voulant que la sévérité des peines soit dissuasive; on sait maintenant que l'interdiction pénale et la peine ont peu ou pas d'influence sur les comportements. On serait maintenant aux prises avec une nouvelle tentative de mystification; la criminalité est en baisse, et ce succès est revendiqué par plusieurs groupes d'intérêt, la police, les législateurs, les partis au pouvoir (Brodeur, 1999). Quelques criminologues seraient même tombés dans le panneau voulant croire ou faire croire que la discipline est pour quelque chose dans cette décrue de la criminalité officielle, mais la majorité se hâte d'invoquer la vraie raison de cette décroissance, à savoir l'importance relative du groupe d'âge connu pour sa propension au crime violent.

Larrivée au gouvernement du Parti conservateur a tout à voir avec le retrait de l'État des subventions à la recherche en criminologie. Les signes d'un changement de fond par rapport aux politiques des Libéraux s'accumulent: le gouvernement conservateur abolit en 2006 la Commission du droit du Canada, les services d'évaluation de la recherche à Condition féminine Canada, l'aide fédérale aux maisons pour femmes violentées, le programme de «contestation judiciaire», c'est-à-dire l'aide financière de l'État fédéral aux plaignants qui en prétendent que les décisions judiciaires ne respectent pas la Charte canadienne des droits et 
libertés. Les membres du cabinet entendent se satisfaire de leurs propres lumières. S'agissant par exemple de la Commission du droit, le ministre de la Justice lui retire sa subvention, il utilisera désormais le budget de cet organisme (trois millions de dollars par année) au paiement d'honoraires aux bureaux d'avocats de son choix lorsque se présenteront des sujets épineux.

Le constat établi en 1999 par les chercheurs canadiens est plutôt sévère; des facteurs externes sont invoqués, mais l'autocritique commence à se manifester. C'est cependant dans une livraison ultérieure de la Revue canadienne de criminologie, devenue entre-temps la Revue canadienne de criminologie et de justice pénale (un changement significatif), que d'autres criminologues canadiens et dans certains cas les mêmes s'interrogent sur la place de la pensée critique dans la discipline.

\section{La place de la pensée critique (2006)}

Sur la place de la perspective critique dans la criminologie canadienne, que lit-on dans le numéro thématique de 2006 de la Revue canadienne de criminologie et de justice pénale? (48 [5]). Cette livraison s'est donné pour titre en français «Droit, crime et critique au Canada», en anglais «Law, Society and Critique in Canada». Le premier contient le mot «crime» qui est omis en anglais. Le mot «Society» est omis en français. Les termes communs aux deux sous-titres sont critique et droit. Ce projet de numéro thématique a été élaboré dans des colloques et les auteurs se sont entendus sur une des causes du malaise qu'ils vont décrire: l'ethos néo-libéral domine à la fois la pensée et les conditions de production intellectuelle en criminologie comme ailleurs depuis quelques années. En criminologie, les projets de recherche s'inspirant d'une perspective critique sont mal reçus par les organismes subventionnaires de la recherche; le chercheur identifié à ce courant peut même se voir interdire l'accès aux données et, s'il arrive à dépasser ces deux premiers obstacles et à mener les études projetées, il ne trouvera pas facilement de périodiques et de maisons d'édition qui voudront en publier les résultats. Dans le chapitre d'introduction, les directeurs de cette livraison, oscillant entre espoir et découragement comme les précédents qui ont contribué au bilan de la recherche en 1999 avouent qu'ils osent rêver à l'avènement d'une «théorie unifiée de la justice sociale», mais reconnaissent que tout ou presque s'y oppose dans le climat actuel, y compris le parti au pouvoir au fédéral. Ils tentent de se 
rassurer en notant que la criminologie diversifie ses perspectives et ses méthodes en recourant à des techniques développées dans d'autres traditions (Martel et al., 2006: 642, traduction libre). Cet espoir de solution par la diversité me paraît aussi mal fondé que celui des féministes qui parlent d'approches duales en recherche.

Apparaissent dans ce bilan des signes de lassitude et l'envie d'un «ailleurs» théorique. Les directeurs d'édition de ce numéro en sont conscients, mais tentent de se rassurer : en cette ère de modernité avancée, les «trans» (transdisciplinarité, trans-courants, etc.) annoncent un renouveau, car ces approches auraient des vertus immanentes. Il faut leur demander: est-ce suffisant? À mon avis, rien n'est moins sûr. Si le passé est garant de l'avenir, même la possibilité d'une pensée critique sur le crime est loin d'être assurée. Comme le remarquent plusieurs auteurs, les efforts antérieurs pour contrer le positivisme juridique et le néo-positivisme clinique ont échoué malgré la ferveur de ceux qui les portaient, Robert Ratner, Brian McLean, John McMullan, trois hérauts de la critique radicale.

Pour ce qui est des féministes, elle trouvent à se rassurer comme c'était le cas dans le bilan de 1999; les travaux inspirés de leur perspective réussissent à faire apparaître les aspects cachés du traitement judiciaire de la violence conjugale, à démystifier les thèses «masculinistes» qui veulent que la violence faite aux hommes égale ou dépasse celle faite aux femmes (Minaker et Snider, 2006), à déconstruire également les fausses accusations d'infanticide portées contre les femmes (Kramar, 2006), à faire la critique des conditions d'emprisonnement des femmes autochtones qu'on place en isolement complet (Martel, 2006).

Au contraire de cet optimisme, c'est le découragement ou presque qui marque l'article cosigné par Dorothy Chunn et Robert Menzies (2006). Pour ces deux auteurs dont on se souviendra qu'ils étaient parmi les optimistes dans le bilan sur la recherche en 1999, les années 2001-2004 ont été particulièrement difficiles; penser «critiquement» en criminologie est devenu impossible. On assiste «à la renaissance des paradigmes de la répression» logés «sous des bannières dites novatrices comme la gestion du risque, la criminologie computationnelle, la criminologie administrative et la cartographie de la criminalité» (Chunn et Menzies, 2006: 663, traduction libre). En somme, une nouvelle orthodoxie est née, la bonne gestion, à laquelle il n'est pas facile de résister. Dans cet article dont le sous-titre est «Surviving the Restructuring of the Discipline in the 21st century», les deux auteurs sont très 
pessimistes. Ils ne sont pas les seuls. Hogeveen et Woolford vont dans le même sens: pour eux, la pratique universitaire a sombré dans la complicité administrative (2006: 682): ils appellent de leurs vœux une critique visant à déstabiliser le statu quo. On a suffisamment fait l'évaluation des structures et des institutions en place, il est temps de passer à autre chose, écrivent-ils. Cependant, leur proposition va dans trop de directions, empruntant tantôt à la phénoménologie, tantôt à la déconstruction chez Derrida, tantôt aux perspectives critiques des années 1970 et 1980. Williams et Lippert (2006) estiment qu'on a trop mis l'accent sur l'État et le pouvoir légal, il est temps d'analyser les formes d'exercice de la gouvernance hors la loi, par exemple en matière d'immigration et d'économie (2006: 703-719).

Ce numéro thématique est fait de contributions trop hétéroclites pour déboucher sur un projet commun. Cependant, dans l'ensemble, on peut y lire, sinon une volonté, du moins un désir de repositionnement de la discipline.

\section{Un mouvement en direction des Social Justice Studies}

L'essentiel se trouve dans l'introduction de ce numéro spécial de la Revue canadienne de criminologie et de justice pénale. En effet, les directeurs d'édition y avouent avoir été inspirés par le dialogue en ligne créé par la Division «criminologie critique» de la Société américaine de criminologie. Les préoccupations de ce groupe ont été marquées par des questions en apparence sémantiques, mais visant plus loin comme on le verra. Un universitaire états-unien dont le département voulait se donner un nouveau nom et passer de «Criminal Justice» à « Social Justice», cherchait auprès de ses collègues ayant de l'expérience en la matière des moyens de défendre la nouvelle appellation devant les autorités administratives de l'établissement. L'échange a montré qu'un vent de changement soufflait sur la «pluridiscipline» qu'est la criminologie.

La majorité des participants était favorable au changement de nom; dans plusieurs milieux, c'était déjà chose faite. L'intitulé "Criminal Justice» datait des années 1960, de l'époque où il était urgent d'entreprendre ce qu'on appelait «la guerre au crime». Parmi les nouvelles appellations, on trouve «Études sur le droit et la justice», "Changement social et développement», «Justice et recherche sociale», «Études sur la criminologie, la justice et les politiques sociales» (voir Martel et al., 2006: 634-635, traduction libre). Dans ces intitulés, le terme «justice» 
est celui qui revient le plus souvent. Le mot «criminologie» apparaît plus rarement et est alors associé à celui de «justice». Cette tendance s'est déjà fait sentir dans la criminologie canadienne, puisque l'Association canadienne de criminologie s'appelle maintenant Association canadienne de criminologie et de justice pénale, et la revue a fait le même ajout qui a le mérite de rappeler que la criminologie n'est pas qu'un ensemble de savoirs, mais aussi une pratique nécessaire à l'élaboration de politiques justes.

Autant je me réjouis de cette addition, autant je continue de croire qu'il faut travailler non pas en aval du droit pénal, c'est-à-dire dans ses conséquences sur les criminalisés, mais en amont, par la déconstruction de la notion juridique de crime, dont il faut faire apparaître les limites. Le législateur n'est pas capable de nommer les valeurs sociales consensuelles au nom desquelles il est légitime d'interdire les comportements, le citoyen doit déduire leur importance de la durée des sentences imposées ou de la procédure! C'est ce genre de critique que formulent la Commission du droit du Canada et un groupe de chercheurs britanniques et irlandais.

\section{En Grande-Bretagne et en Irlande}

Dans un ouvrage récent intitulé Beyond Criminology: Taking Harm Seriously (Hillyard et al., 2004), des collègues britanniques et irlandais qui travaillent depuis plusieurs années dans le champ de la criminologie émettent un diagnostic sévère, voire choquant, sur l'état de la discipline et des connaissances qu'elle génère depuis la fin des années 1970. Voici, résumé en quelques lignes, le problème comme ils le voient:

La criminologie s'est éloignée à tort des considérations épistémologiques et sociopolitiques qui devraient la nourrir pour se tourner vers ses vieilles lunes empiricistes, se réduisant du coup au statut de science appliquée, se nourrissant des problèmes du jour et se laissant vider de sa substance par l'agenda des financiers. (Hillyard et al., 2004: 10. Ma traduction)

Ce bilan fait suite à un travail d'analyse qui s'étend sur plusieurs années, il est inspiré par les "social justice studies», et prend racine dans un mouvement au nom étrange dont il sera question plus loin, la zémiologie.

Mais que signifie «s'éloigner des considérations épistémologiques»? Si l'on en croit les auteurs, les criminologues contemporains auraient 
négligé, depuis plus de trente ans ${ }^{10}$, de faire l'examen de l'objet de la discipline, le crime; ils auraient oublié que ce concept a été emprunté au droit sans critique, et que son contenu est le produit de décisions souvent contradictoires de parlements rarement désintéressés. Les criminologues se seraient tenus loin des théories de la connaissance sur le droit, alors que celles-ci leur auraient permis de comprendre comment s'élabore la norme pénale et à quels intérêts elle répond ${ }^{11}$. Ainsi, «s'éloigner des considérations épistémologiques et sociopolitiques», c'est contribuer à l'hétéronomie de la discipline, alors que travailler à son autonomie exige un effort d'appropriation de son objet, ce que devrait faire la criminologie contemporaine. Il faudra donc déconstruire la notion de crime qu'impose le droit positif, la re-construire et/ou se donner un objet propre et s'assurer de sa valeur heuristique.

Qui sont les auteurs de ce diagnostic? Quelles sont les leçons à tirer de leurs constats? La criminologie canadienne se retrouve-t-elle dans leurs propos?

La particularité de la démarche des collègues d'outre-Atlantique est la suivante: c'est en réfléchissant sérieusement sur et à l'objet de la criminologie, le crime, qu'ils sont arrivés à la conviction qu'il fallait modifier le contenu de la discipline, le sens et la portée du sujet d'étude. Lobjet «crime» défini dans le code pénal leur est apparu comme trop étroit, et le législateur quand il crée ou abolit des interdits ne se soucie pas de l'intérêt général. Les crimes, les délits que retient le droit pénal ne sont pas, de loin, les seuls torts qu'il faut «contenir», ni les plus graves ni les plus dommageables, ni ceux qui font le plus grand nombre de victimes. Les crimes dont parle le droit pénal n'impliquent souvent que deux personnes, un infracteur et sa victime. Les auteurs proposent de s'intéresser plutôt aux torts sociaux graves qui ont en commun de heurter de front les valeurs les plus chères au groupe social, à la nation. Non seulement les codes pénaux ne savent pas parler de ces actes de

10. Les auteurs font ici allusion à deux ouvrages majeurs produits par leurs compatriotes I. Taylor, P. Walton et J. Young, The New Criminology (1973) et Critical Criminology (1975), et aussi à la critique marxiste du droit et du crime qui se trouvait une voix dans la revue de l'École de criminologie de Berkeley, Issues in Criminology, à la même époque.

11. Il existe bien sûr une philosophie, une sociologie et une anthropologie du droit qui éclairent la construction de la norme; il existe aussi une science du droit dont parle éloquemment Vittorio Villa entre autres. Cet auteur fait une distinction utile entre deux épistémologies juridiques, le descriptivisme et le constructivisme en droit, qu'il serait intéressant d'appliquer au droit pénal. Voir son chapitre sur ce sujet, «Entre descriptivisme et constructivisme» dans Théorie du droit et science (LGDJ, 1994 : 288-291). 
malfaisance grave, mais ils conservent dans la liste des conduites prohibées des infractions désuètes, des offenses à la moralité religieuse, alors que le consensus social évolue et n'a que faire de ces vieilles lunes. Enfin, le législateur fait passer dans le droit pénal des valeurs de classe, de genre et d'origine ethnique, et il lui arrive aussi d'ajouter des interdits sous la pression de l'opinion publique réagissant à des événements tragiques mais uniques, sans égard à l'intérêt général.

Quels sont ces grands torts sociaux qui n'ont pas trouvé place dans le code pénal? Ce sont les crimes de l'État lui-même (Ward, 2004: 84-100), par exemple la négligence des gouvernants lors de grands conflits et de désastres, leurs omissions; celles des professions comme la médecine, le génie et le droit, qui ne savent pas respecter leur code d'éthique et se soucient peu du bien commun; c'est l'indifférence morale des puissants et des riches, de même que celle, plus incompréhensible et d'ailleurs plus rare, de l'État (Pemberton, 2004: 67-84); ce sont les conditions de travail que maintient la grande industrie, indifférente aux blessures et aux morts qu'elle cause, préférant payer les dommages plutôt que de rendre le milieu de travail sécuritaire et agréable (Tombs, 2004: 156-178); ce sont les crimes des grandes corporations qui violent les règles du jeu économique; c'est l'incapacité de l'État d'appliquer ses propres règles et de s'auto-sanctionner quand il triche et trompe les citoyens $^{12}$. Ce sont ces torts que les auteurs de Beyond Criminology voudraient sortir de l'ombre, nommer, rendre contrôlables et tenter de contenir. Mais par quels moyens? Cela est moins clair dans le programme qu'ils proposent. Sans doute en faisant appel parfois au droit civil et aux sanctions économiques, en recourant le plus possible aux pratiques de la justice restauratrice, aux mesures de réparation, à la médiation, appuyées, parfois, par la sanction pénale.

\section{La zémiologie}

En somme, la criminologie a commis une erreur de fond en empruntant son objet au droit pénal et en s'y limitant sans en faire la critique. Les sciences humaines et sociales ont autre chose à faire qui ressortit mieux à leur compétence. Elles doivent s'intéresser aux méfaits qui gâchent la

12. Il faut reconnaître cependant qu'avec ses règles de procédure, le droit est incapable de sanctionner ces grandes malfaisances, car il s'est contraint lui-même à n'inculper que ceux dont il peut démontrer l'intention coupable et la responsabilité individuelle. 
vie de pans entiers du groupe social ${ }^{13}$. Cette discipline si elle vient au jour s'appellera zémiologie, du grec zemia, qui veut dire «barm» en anglais, «tort» en français. C’est en février 1999 qu'est né, dans le Devon, un mouvement qui s'est nommé lui-même «zemiology». Le but avoué de la conférence qui a réuni les collègues britanniques et irlandais était d'explorer la possibilité de dépasser l'analyse du crime. L'argumentaire $\mathrm{du}$ groupe fondateur est le suivant: au cours de leur vie, les personnes humaines seront soumises à des torts sociaux et physiques, à la maison, dans la rue, au travail et dans leurs loisirs. Ces torts sont la pauvreté, la malnutrition, la guerre, la violence, la pollution, les accidents de la route, les maladies, le crime, la négligence des professionnels de la santé et les catastrophes naturelles. Plusieurs de ces torts sont reconnus et pris en charge par des organismes publics, la police, les services de santé, les services sociaux ou les tribunaux de droit civil à la suite des plaintes des victimes. Mais progressivement, à notre époque néo-libérale, l'État se retire des domaines d'intervention qui lui créent des soucis et ne contribuent pas à sa popularité, et ce sont des agences privées qui prennent ou tentent de prendre le relais. D'autres malfaisances ne sont pas connues et donc pas reconnues hors de l'expérience douloureuse de ceux qui les subissent. Ceux-ci, trop souvent des mal nantis, ne savent pas nommer ce qui les agresse ou les handicape à vie. Ils croient que les malheurs ou la malchance leur tombent dessus, qu'il n'y a rien à faire. Ils ne savent pas réclamer réparation, ni proposer des changements sociaux. Une question s'impose ici: les zémiologues vont-ils pouvoir s'entendre sur la notion de tort? Va-t-on se perdre dans les problèmes de définition?

Pour comprendre l'orientation que prend le mouvement, il est nécessaire de voir qui en sont les inspirateurs et comment s'est forgé leur programme de justice sociale. Paddy Hillyard était, en 1999, directeur du Centre d'étude sur l'exclusion sociale et la justice sociale à l'Université de Bristol, et David Gordon, directeur du Centre Townsend d'étude sur la pauvreté internationale, également à l'Université de Bristol. Les autres participants provenaient des universités de Liverpool, de Sunderland, de Belfast, etc. S'y sont ajoutées des personnalités extérieures au monde universitaire, un économiste de la Banque mondiale, et un membre du comité britannique des Nations Unies sur l'environnement.

13. L'expression est la mienne. Je crois que les auteurs ne l'utilisent qu'une fois dans le cadre de leur ouvrage collectif. 
Leurs premiers échanges portaient sur la violence dans les sociétés démocratiques, les catastrophes naturelles, la responsabilité criminelle corporative, la "carte» des grands torts sociaux, la santé au travail, les morts causées par les accidents au travail et les institutions génératrices de tort (Hillyard et al., 2004). Le groupe s'est aussi intéressé à la criminalité du pouvoir politique, notant que l'État central ou national n'est pas le seul en cause: les pouvoirs régionaux et municipaux sont eux aussi les témoins passifs de la création de zones de pauvreté, ils participent à la structuration et à la délimitation de ces zones «dangereuses» afin de préserver la valeur immobilière d'autres quartiers. Un géographe montrait que les deux variables associées le plus étroitement aux homicides sont liées au lieu où se trouve la victime et au degré de détérioration des immeubles et de l'espace public environnants plutôt qu'au modus operandi de l'agresseur et à la personnalité de la victime (Dorling, 2006). La détérioration des lieux à laquelle le pouvoir municipal collabore parfois en n'investissant que dans les quartiers avantagés, contribue à rendre criminogènes les quartiers pauvres.

Le diagnostic que posent nos collègues britanniques et irlandais quant aux limites de la criminologie n'est pas sans parenté avec les critiques que lui adresse au même moment la criminologie canadienne dans les deux numéros thématiques de la Revue canadienne de criminologie sur la recherche $\left(1999^{14}\right)$ et sur la pensée critique $\left(2006^{15}\right)$. Comme on l'a vu, la plupart des auteurs canadiens avouent qu'il est devenu très difficile de penser «critiquement» sur la question pénale au Canada (Woolford et al., 2006). Mais cette excuse n'est-elle pas trop facile? Comment expliquer que dans un climat économique et politique comparable, des criminologues britanniques et irlandais aient réussi à organiser la résistance et à formuler une critique radicale de l'objet de la criminologie? C'est dans l'Angleterre de Tony Blair que le groupe proposant la zémiologie a commencé à se mobiliser en 1999. Mais les collègues qui en ont pris l'initiative travaillent depuis quelques années déjà à élargir l'objet de leurs préoccupations depuis des centres d'étude

14. Revue canadienne de criminologie. La recherche criminologique et la justice pénale au Canada, Tendances actuelles et perspectives d'avenir (sous la direction de Chris Murphy et de Philip Stenning), 41 (2), 127-330. Le numéro comprend dix-neuf contributions, certaines signées par plus d'un auteur.

15. Revue canadienne de criminologie et de justice pénale. Law, Society and Critique in Canadal Droit, crime et pensée critique au Canada (sous la direction de Bryan Hogeveen, Joane Martel et Andrew Woolford), 48 (5), 631-836. 
sur la pauvreté, l'exclusion sociale et la justice sociale, en amont de la criminalisation. Ils sont prêts à rompre avec la criminologie.

Rompre avec la discipline mère est plus difficile au Canada, pays où la criminologie s'est développée comme une profession dans quatre grandes universités (Montréal, Toronto, Ottawa et Simon Fraser) et même dans quelques départements de sociologie; elle est devenue en quelque sorte une machine à produire des diplômés du premier cycle qui pratiquent une gestion des cas ordonnée par l'appareil pénal.

\section{"Qu'est-ce qu'un crime? " Les travaux de la Commission du droit du Canada}

De 2001 à 2006, la Commission du droit du Canada a contribué de façon importante à la transformation du paradigme de la criminologie. Elle a lancé une série de travaux qui ont débuté par un débat en ligne auxquels se sont joints les criminologues et les pénologues d'une vingtaine de pays. La question posée était «Qu'est-ce qu'un crime?». Un document de travail portant le même titre a inspiré des colloques et des tables rondes, autour des questions: "Quelles sont les conduites qui apparaissent franchement indésirables aux yeux des Canadiens?»; «N'a-t-on pas trop souvent tendance à recourir au droit pénal pour régler nos conflits?»; «N'existe-t-il pas, pour la plupart des cas, des recours plus conformes à nos valeurs démocratiques que la sanction pénale?».

Trois études commanditées par la Commission démontrent les limites du droit pénal, la notion de crime n'a ni utilité ni application dans les domaines de la fraude à l'assurance, de la protection de l'environnement, de la délinquance informatique et du droit d'auteur. Dans d'autres domaines, le recours au pénal a des effets douteux, moralement, éthiquement et même politiquement, comme dans le contrôle des armes à feu et la sécurité nationale. Dans ce dernier cas, l'application de la loi a des conséquences carrément discriminatoires sur les immigrants et les réfugiés. Par ailleurs, la légalisation des jeux de hasard montre comment l'État a su interdire, au nom de raisons morales, cette activité pendant plusieurs décennies, pour ensuite la déclarer légale, en faire la promotion et en tirer des avantages financiers considérables (Brodeur, 2004).

Les recherches effectuées dans le cadre de la Commission du droit du Canada démontrent, si besoin était, que le droit pénal n'est ni neutre ni vertueux par nature. Il est trop souvent appelé à jouer un rôle qui 
dépasse sa compétence. Il lui arrive de pratiquer la délinquance et de violer ses propres normes. C'est assez dire qu'il faut user de cette forme de régulation des conduites avec prudence et modération. La criminologie canadienne n'est pas aussi attentive qu'il le faudrait aux signes de dérives du système pénal, ni aussi engagée qu'on le souhaiterait dans une action énergique et concertée contre les dénis de justice les plus flagrants - on pense à l'affaire Walkerton ou à celle du sang contaminé où le droit pénal et sa procédure montrent bien leurs limites.

Le deuxième recueil d'études de cas viendra compléter ces premiers travaux avec des exemples encore plus convaincants des applications iniques auxquelles se prête le droit pénal dans le traitement des conflits sociaux, ethniques et politiques.

\section{Conclusion}

J'ai voulu, dans un premier temps, situer la criminologie canadienne et celle de Montréal dans leur histoire et dans le contexte plus large des sciences sociales et de leur évolution, afin de faire apparaître les obstacles à la connaissance critique en sciences humaines appliquées, et surtout en criminologie, science normative s'il en est. Il m'apparaît que le principal obstacle à la pensée critique est la professionnalisation de la discipline qui en fait une complice du pouvoir pénal. Il est bien difficile, quand on forme de futurs employés de l'État, de penser librement à son objet d'étude sans scier la branche sur laquelle on est assis.

Quant à mon hypothèse voulant que l'absence de réflexion systématique sur cet objet soit largement responsable des apories de la criminologie, elle est partagée par bon nombre des auteurs d'études recensées dans le bilan sur les perspectives critiques en criminologie (2006), mais elle l'est davantage par la Commission du droit du Canada et par les collègues britanniques et irlandais. Il faut élargir le champ d'étude et son application. Cette ambition est-elle réalisable? Oui, à mon avis. Les travaux de la Commission du droit du Canada montrent la possibilité d'approches autres que pénales dans le règlement des conflits, par exemple le recours accru au droit civil, aux sanctions économiques, à la médiation, à la réparation. De leur côté, les zémiologues sont "passés à l'acte» si on peut dire, ils ont élaboré des stratégies de recherche élargissant considérablement la mission de leurs propres centres de recherche qui portent maintenant sur la pauvreté, l'exclusion et la justice sociales. 
Ce besoin d'extension du champ d'étude en amont du crime et de la criminalisation se manifeste aussi dans les «Justice Studies», un changement d'appellation désiré par les membres de la Division de la criminologie critique de l'American Society of Criminology. Certes, les indices de changement sont fragiles. Les Justice Studies et la zémiologie sont des projets marginaux dans leurs milieux respectifs. Les propositions des zémiologues sont bien vastes et les objectifs visés trop larges. Cependant, la graine est semée, et tôt ou tard elle peut germer. La criminologie traditionnelle s'est déjà laissée ébranler par la théorie de la réaction sociale, l'interactionnisme symbolique, le constructivisme, le féminisme et la critique du racisme. Ce n'est pas la première fois qu'elle fait son autocritique. Il y a 70 ans, Edwin Sutherland (1949) s'opposait à Paul Tappan, qui ne voulait voir de tort et de mal que dans ce que le législateur définissait comme crime; les vrais crimes, disait-il, ne sont pas dans le code pénal et y seraient-ils que la procédure pénale ne permettrait pas de les sanctionner. Les criminels en col blanc, les chefs d'industrie, les grands financiers de la planète et même les hommes d'État (moins que les précédents) sont capables de tricherie, de mensonge, de cruauté, mais ils appartiennent à cet «upperworld» dont la position sociale, l'intelligence et l'habileté criminelle permettent de se mouvoir parmi leurs concitoyens « immune to recognition and prosecution as criminals» (Sutherland, 1983: xxxi).

On ne saurait mieux dire. Ce sont là les deux «faiblesses du crime», il ne sait pas parler des grands torts, et l'appareil pénal ne sait pas se saisir des puissants. Sur ces grands problèmes, la criminologie canadienne est loin d'avoir fait l'impasse, comme le montre bien le bilan de 2006, sur la place de la critique dans la criminologie canadienne. Et au-delà du discours, on voit apparaître des programmes d'action dans les travaux de la Commission du droit comme dans ceux de nos collègues d'outre Atlantique, et une nouvelle orientation dans les Justice Studies. Ce sont là de très bonnes nouvelles.

\section{Références}

Bertrand, M.-A. (1967). The Myth of Sexual Equality before the Law. In Actes du $5^{e}$ Colloque de recherche sur la délinquance et la criminalité (129-161). Montréal: Société de criminologie du Québec.

Bertrand, M.-A. (1986). Perspectives traditionnelles et perspectives critiques en criminologie. Criminologie, XIX (1), 97-111. 
Bertrand, M.-A. (Ed.) (1998). Prisons pour femmes. Montréal: Les Éditions du Méridien.

Bertrand, M.-A. (Ed.) (1999a). Genre, hétérosexualité, ethnicité et norme. Revue canadienne droit et société / Canadian Journal of Law and Society, 14 (1).

Bertrand, M-.A. (1999b). Incarceration as a Gendering Strategy. Revue canadienne droit et société / Canadian Journal of Law and Society, 14 (1), 45-60.

Bertrand, M.-A. (2000). L'état de la pensée critique sur le racisme en criminologie. In M. Salmador Louis \& P. Poussaint (eds.), Collectif interculturel: La revue de l'Institut de recherche et de formation interculturelles de Québec, IV (2), 37-48.

Bertrand, M.-A. (2003). Les femmes et la criminalité. Montréal: Les Éditions Athéna.

Bertrand, M.-A. (2004). Les effets du genre et de la couleur du droit sur les femmes autochtones judiciarisées. In J. Poupart (ed.), Au-delà du système pénal (169-190). Québec: Presses de l'Université du Québec.

Brodeur, J.-P. (1983). High Policing and Low Policing: Remarks about the Policing of Political Activities. Social Problems, 20 (5), 507-520.

Brodeur, J.-P. (1986). Provocations. Criminologie, XIX (1), 141-169.

Brodeur, J.-P. (1999). Disenchanted criminology. Canadian Journal of Criminology / Revue canadienne de criminologie, 41 (2), 131-136.

Brodeur, J.-P. (2004). What is a crime? A Secular Answer. In Law Commission of Canada (1-33). Vancouver: UBC Press.

Chunn, D. E., \& Menzies, R. (2006). So what does all this have to do with criminology?: Surviving the Restructuring of the Discipline in the TwentyFirst Century. Canadian Journal of Criminology and Criminal Justice / Revue canadienne de criminologie et de justice pénale, 48 (5), 663-680.

Clairmont, D. (1999). In defence of liberal models of research and policy. Canadian Journal of Criminology / Revue canadienne de criminologie, 41 (2), 151159.

Clark, B. C. (1998). Creating Entrepreneurial Universities: Organizational pathways of Transformation. Oxford: Pergamon / Elsevier Science.

Comack, E. (1999). New possibilities for a feminism «in» criminology? From dualism to diversity. Canadian Journal of Criminology / Revue canadienne de criminologie, 41 (2), 161-170.

De Lint, W. (2006). Governmentality, Critical Criminology, and the Absent Norm. Canadian Journal of Criminology and Criminal Justice / Revue canadienne de criminologie et de justice pénale, 48 (5), 721-734.

Doob, A. (1999). Youth justice research in Canada: An assessment. Canadian Journal of Criminology / Revue canadienne de criminologie, 41 (2), 217-224.

Dorling, D. (2006). Prime Suspect: Murder in Britain. Prison Service Journal, 166, 3-10.

Fournier, M. (1998). Entretiens avec Denis Szabo: Fondations et fondements de la criminologie. Montréal: Éditions Liber.

Geiger, J.-L. (2004). Knowledge and Money. Stanford: Stanford University Press. 
Hillyard, P., Pantazis, C., Tombs, S., \& Gordon, D. (2004). Beyond Criminology: Taking Harm Seriously. London: Pluto Press.

Hogeveen, B., \& Woolford, A. (2006). Critical Criminology and Possibility en the Neo-liberal Ethos. Canadian Journal of Criminology and Criminal Justice / Revue canadienne de criminologie et de justice pénale, 48 (5), 681-702.

Jaccoud, M. (Ed.) (2003). La justice réparatrice et la médiation: convergences ou divergences. Paris: Éditions L'Harmattan.

Kramar, K. (2006). Coroners' Interested Advocacy: Understanding Wrongful Accusations and Convictions. Canadian Journal of Criminology and Criminal Justice / Revue canadienne de criminologie et de justice pénale, 48 (5), 803-821.

Landreville, P. (1986). Évolution théorique en criminologie, histoire d'un cheminement. Criminologie, XIX (1), 11-31.

Laprairie, C. (1999). The impact of aboriginal justice research on policy: A marginal past and an even more uncertain future. Canadian Journal of Criminology / Revue canadienne de criminologie, 41 (2), 249-260.

Law Commission of Canada (2005). What is a Crime? Defining Criminal Conduct in Contemporary Society. Toronto / Vancouver: UBC Press.

Lemire, G. (1986). Théories et pratiques criminologiques: quand l'organisation mène le jeu. Criminologie, XIX (1), 215-226.

Martel, J. (2006). Les femmes et l'isolement cellulaire au Canada: un défi de l'esprit sur la matière. Canadian Journal of Criminology and Criminal Justice / Revue canadienne de criminologie et de justice pénale, 48 (5), 781-801.

Martel, J., Hogeveen, B., \& Woolford, A. (2006). The State of Critical Scholarship in Criminology and Socio-Legal Studies in Canada. Canadian Journal of Criminology and Criminal Justice / Revue canadienne de criminologie et de justice pénale, 48 (5), 633-646.

Menzies, R., \& Chunn, D. E. (1999). Discipline in Dissent: Canadian academic criminology at the millennium. Canadian Journal of Criminology / Revue canadienne de criminologie, 41 (2), 285-297.

Minaker, J. C., \& Snider, L. (2006). Husband Abuse: Equality with a Vengeance? Canadian Journal of Criminology and Criminal Justice / Revue canadienne de criminologie et de justice pénale, 48 (5), 753-780.

Moore, D., \& Rennie, A. (2006). Hated Identities: Queers and Canadian Antihate Legislation. Canadian Journal of Criminology and Criminal Justice / Revue canadienne de criminologie et de justice pénale, 48 (5), 823-836.

Murphy, C. (1999). The current and future state of police research and policy in Canada. Canadian Journal of Criminology / Revue canadienne de criminologie, 41 (2), 205-215.

Murphy, C., \& Stenning, P. (1999). Introduction. Canadian Journal of Criminology / Revue canadienne de criminologie, 41 (2), 127-130.

Normandeau, A. (1986). Stratégies de changement et politiques pénales. Criminologie, XIX (1), 171-187.

Normandeau, A., \& Cusson, M. (1999). La criminologie au Québec: 19601999. Canadian Journal of Criminology / Revue canadienne de criminologie, 41 (2), 299-309. 
Pemberton, S. (2004). A Theory of Moral Indifference: Understanding the Production of Harm by Capitalist Society. In P. Hillyard, C. Pantazis, D. Gordon \& S. Tombs (eds.), Beyond Criminology: Taking Harm Seriously (67-83). London: Pluto Press.

Roberts, J. (1999). Sentencing research in Canada. Canadian Journal of Criminology / Revue canadienne de criminologie, 41 (2), 225-234.

Smandych, R., \& Hogeveen, B. (1999). On the fragmentation of Canadian criminal justice history. Canadian Journal of Criminology / Revue canadienne de criminologie, 41 (2), 191-203.

Stenning, P. (1999). Implications of Public service reform for criminal justice research and policy in Canada. Canadian Journal of Criminology / Revue canadienne de criminologie, 41 (2), 179-190.

Stenning, P., \& Murphy, C. (1999). Concluding thoughts. Canadian Journal of Criminology / Revue canadienne de criminologie, 41 (2), 321-323.

Sutherland, E. (1949). White Collar Crime. New York: Holt Rinehart and Winston.

Sutherland, E. (1983). White Collar Crime. The Uncut Version. New Haven: Yale University Press.

Taylor, I., Walton, P., \& Young, J. (1973). The New Criminology: For a Social Theory of Deviance. London: Routledge \& Kegan Paul.

Taylor, I., Walton, P., \& Young, J. (1975). Critical Criminology. London: Routledge \& Kegan Paul.

Tombs, S. (2004). Work Place Injury and Death: Social Harm and the Illusion of Law, State Corporations and the Production of Inequality. In P. Hillyard, C. Pantazis, S. Tombs \& D. Gordon (eds.), Beyond Criminology: Taking Harm Seriously (156-177). London: Pluto Press.

Trépanier, J. (1986). La justice des mineurs au Québec: 25 ans de transformations (1960-1985). Criminologie, XIX (1), 189-213.

Villa, V. (1994). La science juridique entre descriptivisme et constructivisme. In Amselek, P., Théorie du droit et science (281-291). Paris: P.U.F.

Ward, T. (2004). State Harms. In P. Hillyard, C. Pantazis, S. Tombs \& D. Gordon (eds.), Beyond Criminology: Taking Harm Seriously (84-100). London: Pluto Press.

Williams, J. W., \& Lippert, R. (2006). Governing on the Margins: Exploring the Contributions of Governmentality Studies to Critical Criminology in Canada. Canadian Journal of Criminology and Criminal Justice / Revue canadienne de criminologie et de justice pénale, 48 (5), 703-719.

Woods, G. (1999). Then and now: Federal support for justice research. Canadian Journal of Criminology/Revue canadienne de criminologie, 41 (2), 171-178.

Wood, J., \& Shearing, C. (1999). Reinventing intellectuals. Canadian Journal of Criminology / Revue canadienne de criminologie, 41 (2), 311-320.

Woolford, A., Hogeveen, B., \& Martel, J. (2006). An Opening / Une ouverture. Canadian Journal of Criminology and Criminal Justice / Revue canadienne de criminologie et de justice pénale, 48 (5), 631-632. 